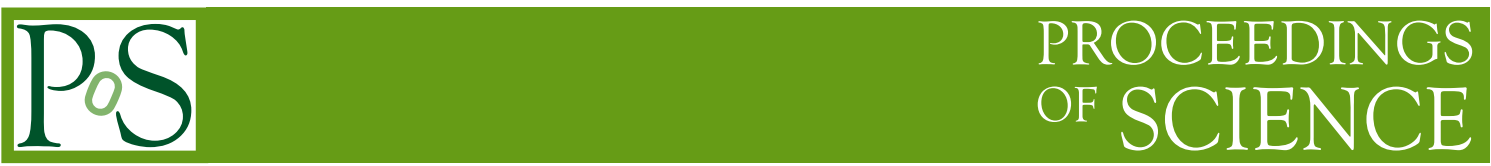

\title{
The Latest Results on High Energy Cosmic Rays
}

\author{
Andrei Kounine*t \\ Massachusetts Institute of Technology, Cambridge, USA \\ E-mail: Andrei.Kounine@cern.ch
}

\section{Samuel Ting}

Massachusetts Institute of Technology, Cambridge, USA

Four detectors operate currently in space exploring a new and exciting frontier in physics research: AMS, CALET, DAMPE, and ISS-CREAM. Of these four detectors AMS is the only magnetic spectrometer. Precision measurements by AMS of the fluxes of cosmic ray positrons, electrons, antiprotons, protons and light nuclei as well as their ratios reveal several unexpected and intriguing features. The presented measurements extend the energy range of the previous observations with much increased precision. The new results show that the positron flux rises from $\sim 10 \mathrm{GeV}$ above the rate expected from cosmic ray collisions with interstellar gas and then exhibits a sharp drop off above $\sim 300 \mathrm{GeV}$. This is consistent with a new source of high energy positrons. Surprisingly, in this rigidity (i.e. momentum divided by charge) range the spectral indices of cosmic ray nuclei experience progressive hardening over the rigidity interval of few hundred GV. This hardening is more pronounced for the secondary nuclei such as lithium, beryllium, and boron than for the primary nuclei helium, carbon and oxygen. Most importantly, AMS continues studies of complex antimatter candidates with stringent detector verification and collection of additional data.

The 39th International Conference on High Energy Physics (ICHEP2018)

4-11 July, 2018

Seoul, Korea

\footnotetext{
* Speaker.

${ }^{\dagger}$ On Behalf of the AMS Collaboration.
} 


\section{Detectors for Cosmic Ray studies}

Interest in space-borne particle physics experiments stems from the unique features of experimentation in space, the possibility of studying primordial particles created in the cosmos in an almost background-free environment. Four detectors operate currently in space exploring a new and exciting frontier in physics research: AMS (since May 2011), CALET (since August 2015), and ISS-CREAM (since August 2017) onboard the International Space Station and DAMPE (since December 2015) as a free-flying satellite. Of the four experiments AMS is the only magnetic spectrometer. This feature makes it unique in studies of cosmic ray antimatter particle such as positrons, antiprotons and complex antimatter nuclei. The improvement in accuracy over previous measurements is due to its long duration time in space, large acceptance, built in redundancy and thorough calibration. Since its installation on the ISS, AMS has collected more than 120 billion cosmic rays.

The layout of the AMS detector [1] is shown in Fig. 1. It consists of 9 planes of precision silicon Tracker; a Transition Radiation Detector, TRD; four planes of Time of Flight counters, TOF; a Magnet; an array of anti-coincidence counters, ACC, surrounding the inner Tracker; a Ring Imaging Čerenkov detector, RICH; and an Electromagnetic Calorimeter, ECAL. The figure also shows a high energy positron of $868 \mathrm{GeV}$ recorded by AMS.

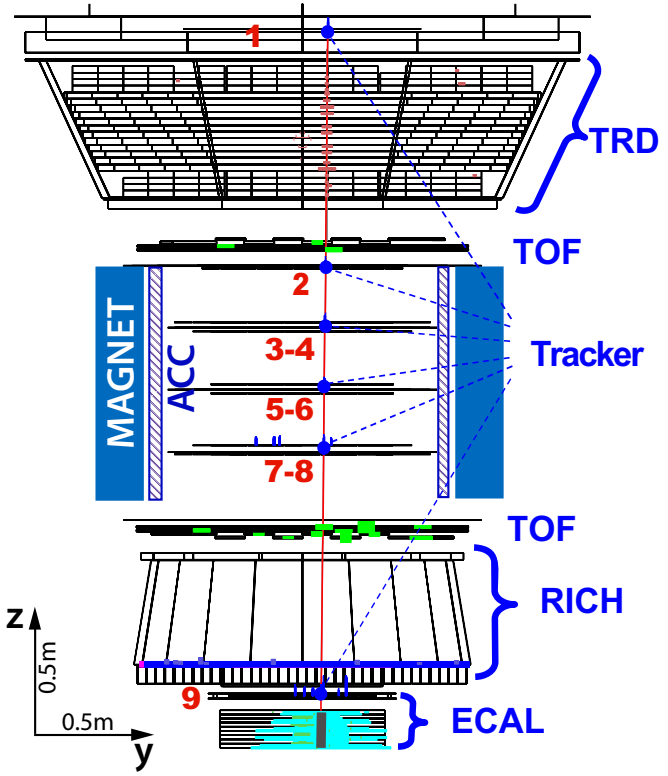

Figure 1: A $868 \mathrm{GeV}$ positron event measured by AMS. Tracker planes 1-9 measure the particle charge, sign and momentum. The TRD identifies the particle as an electron/positron. The TOF measures the charge and ensures that the particle is downward-going. The RICH measures the charge and velocity. The ECAL independently identifies the particle as an electron/positron and measures its energy.

\section{Precision measurement of the positron fraction and fluxes of $\mathrm{e}^{+}$and $\mathrm{e}^{-}$}

Over the past few decades there has been strong interest in the cosmic ray positron fraction in both particle physics and astrophysics [2]. It was considered as a sensitive probe into the properties of Dark Matter in our Galaxy. The first experimental excitement came from earlier experiments [3]. 


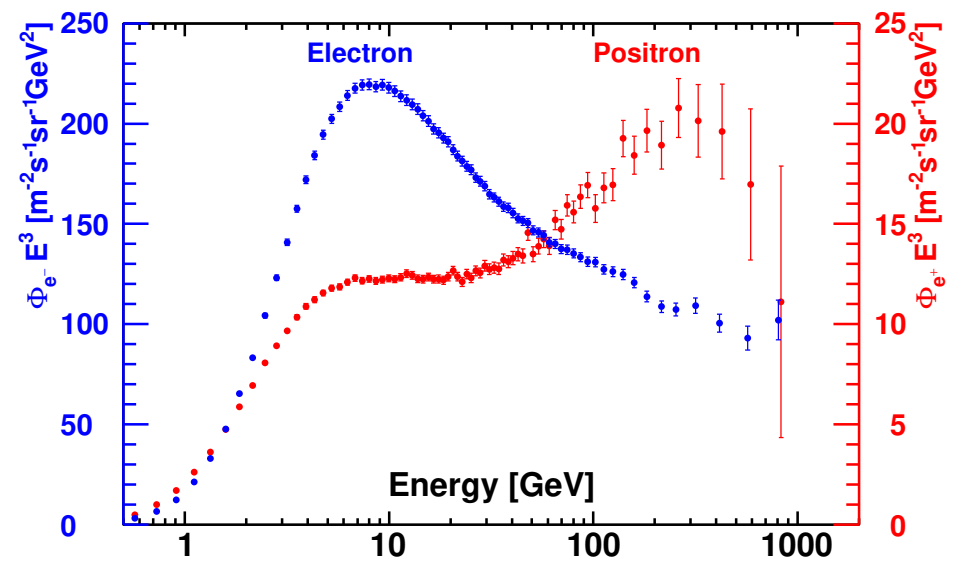

Figure 2: The AMS positron and electron fluxes multiplied by $E^{3}$. The present measurement extends the energy range to $1000 \mathrm{GeV}$ for positrons and electrons.

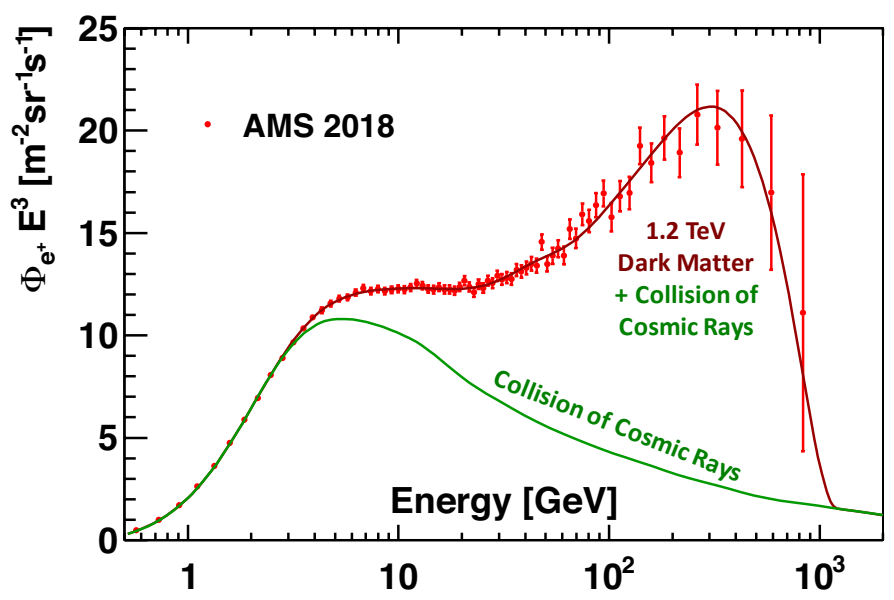

Figure 3: The measured positron spectrum $\left(E^{3} \Phi_{e^{+}}\right)$in comparison with the 1.2 Tev Dark Matter model.

Precision AMS results [4] not only clearly show the rise of the positron fraction above its minimum at $7.8 \mathrm{GeV}$, but also existence of a maximum at $275 \pm 32 \mathrm{GeV}$ [5].

Much more information on the dynamics of this behavior is in the measurements of the individual fluxes of electrons and positrons [6]. To date, AMS has observed that with a data sample of 28.1 million electrons and 1.9 million positrons [6], the electron flux and positron flux display different behaviors in their energy dependences and magnitudes (see Fig. 2). The most striking feature is in the hardening of the positron spectrum starting from $\sim 10 \mathrm{GeV}$, seen as the progressive rise of the positron spectrum (i.e. the flux scaled by $E^{3}, E^{3} \Phi_{e^{+}}$) and its sharp drop off above $\sim 300 \mathrm{GeV}$. This may be interpreted as a contribution of a new, yet unknown, physics source.

The possible relation of Dark Matter annihilation to the positron spectrum has always attracted attention. When particles of dark matter collide, they produce equal amounts of energetic electrons and positrons, which may create structures in the corresponding spectra: an increase with energy followed by a sharp drop off at the mass of dark matter as well as an isotropic distribution of the arrival directions. The expected effect is less pronounced in the abundant cosmic rays like electrons, 
and enhanced in rare species like positrons - exactly as observed by AMS. All the observed features of positron flux are well consistent with a new source of high energy cosmic ray positrons such as the annihilation of Dark Matter particles with a mass of $\sim 1 \mathrm{TeV}$. As seen in Fig. 3, after rising from $\sim 10 \mathrm{GeV}$ above the rate expected from cosmic ray collisions with interstellar gas (green curve), the measured spectrum exhibits a sharp drop off above $\sim 300 \mathrm{GeV}$. With more data AMS will explore this very intriguing feature of the positron flux at high energies.

Following the AMS publications [5, 6], there have been many interpretations [9] with two popular classes. In the first, the excess of $e^{+}$comes from pulsars. In this case, after flattening out with energy the positron spectrum, $E^{3} \Phi_{e^{+}}$, will begin to slowly decrease and a dipole anisotropy should be observed. This scenario is already disfavored following the HAWC observation [10]. In the second, the shape of the positron fraction is due to the annihilation of Dark Matter. In this case, after flattening out, the positron spectrum, $E^{3} \Phi_{e^{+}}$, will decrease rapidly with energy due to the finite and specific Dark Matter mass (see Fig. 3) and no dipole anisotropy will be observed. Over its lifetime, AMS will reach a dipole anisotropy sensitivity of $\delta \simeq 0.01$ at the $95 \%$ C.L. [11].

Other space borne detectors, which do not differentiate between positive and negative charges, measure combined electron and positron flux, $\Phi_{e^{+}+e^{-}}$. Figure 4 shows comparison of the results of CALET and DAMPE presented at the conference [7] together with earlier measurements [8] of ATIC, HESS, and Fermi-LAT. However, as discussed above, these measurements are less sensitive to a small contribution of new sources of high energy electrons and positrons.

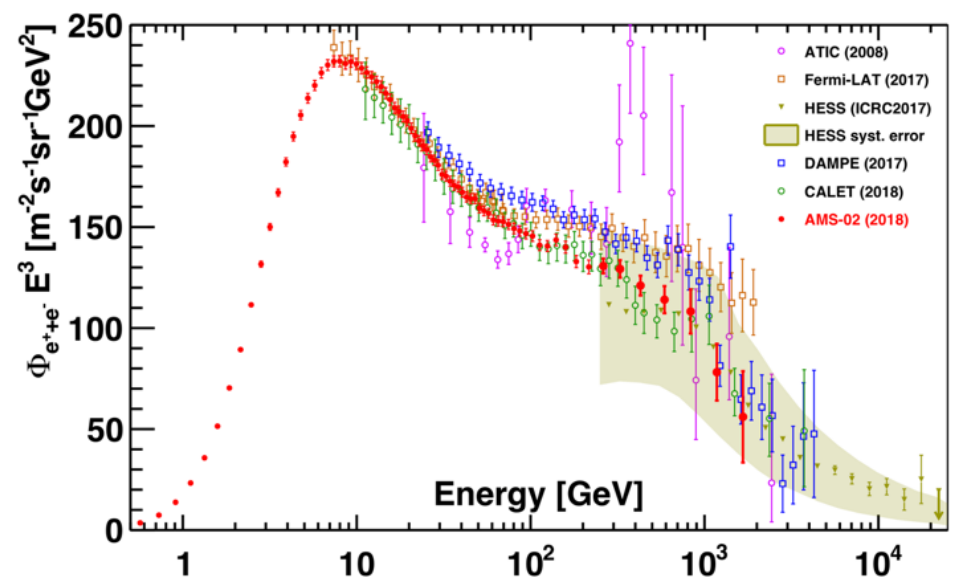

Figure 4: Latest results on the combined positron and electron spectrum $\left(E^{3} \Phi_{e^{+}+e^{-}}\right)$.

\section{Properties of fluxes of elementary particles and their ratios}

Experimental data on the cosmic ray antiprotons, $\bar{p}$, are crucial for understanding the origin of antiprotons in cosmos and for providing an insight into new physics phenomena. While some of cosmic ray $\bar{p}$ are produced by interactions of the cosmic ray nuclei with the interstellar gas, there could be a substantial contribution from annihilation of dark matter particles, acceleration of antiprotons to high energies in astrophysical objects, or evaporation of primordial black holes. The sensitivity of cosmic ray antiprotons to these new phenomena is complementary to the sensitivity of the measurements of cosmic ray positrons $e^{+}$. 


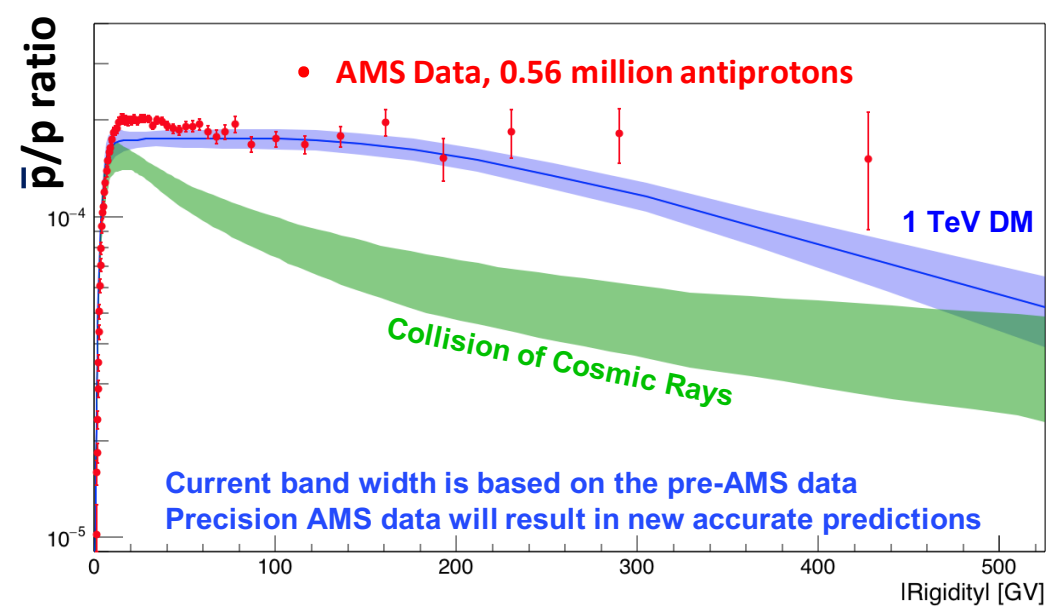

Figure 5: The AMS results on the ratio $\overline{\mathrm{p}} / \mathrm{p}$ in comparison with the model predictions prior to AMS that expected decreasing ratio secondary antiprotons with rigidity [15]. The AMS measurement extends the rigidity range to $525 \mathrm{GV}$ and demonstrates that above $\sim 60 \mathrm{GV}$ the $\overline{\mathrm{p}} / \mathrm{p}$ remains almost flat.
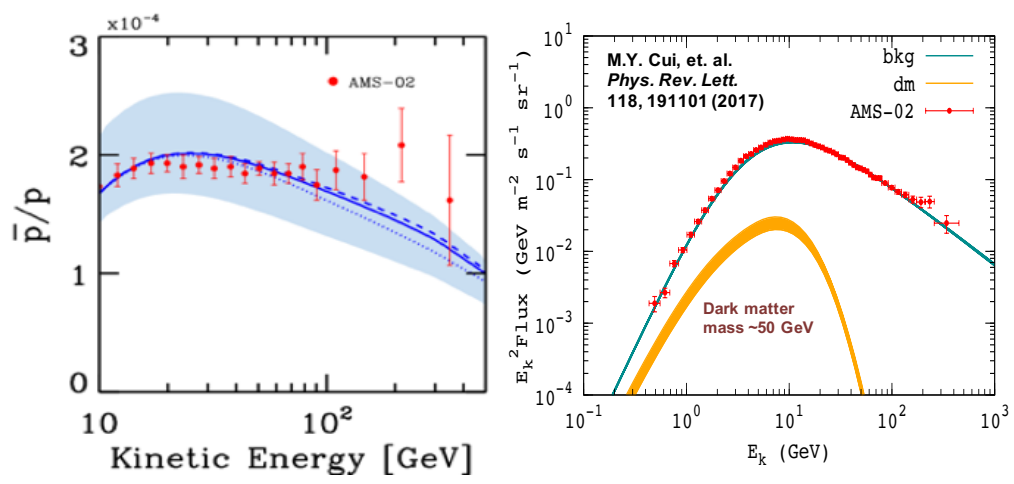

Figure 6: AMS data in comparison with the recent models of secondary antiproton production [16] (left plot) and Dark Matter antiproton production [17] (right plot).

Overall, in the absolute rigidity range $1-525 \mathrm{GV} 5.6 \times 10^{5}$ antiproton events are selected. It is important to note that in the high rigidity range above $100 \mathrm{GV}$ AMS has 2200 events [12]. This can be compared with 3 events detected before AMS [13, 14]. The measured ratio $\overline{\mathrm{p}} / \mathrm{p}$ is presented in Fig. 5 together with model predictions. Above $\sim 60 \mathrm{GeV}$ the ratio is found to be independent on rigidity [12]. This observation caused a major revision of modelling of cosmic ray antiprotons produced in ordinary collisions of protons with interstellar media. This can be seen from comparison of the data with the various models. A model based on earlier data [15] predicts significant deficit of secondary antiprotons compared to the AMS data as seen in Fig. 5, while newer models $[16,17]$ predict more secondary antiprotons above $100 \mathrm{GV}$, as shown in Fig. 6. Still, even revised conservative models (with generously assigned theoretical uncertainties [16]) predict $\sim 50 \%$ drop of the ratio from the maximum, not supported by the AMS data (left plot in Fig. 6), whereas optimistic models (with tight uncertainties for secondary antiproton production) [17] see quite a bit of discrepancies with experimental data, ascribing these discrepancies to the Dark Matter contribution, as illustrated in Fig. 6, right plot. 
It is interesting to compare behavior of the spectra of all elementary particles among themselves. Traditionally, electrons and protons are assumed to be primary cosmic rays, i.e. particles produced directly at sources of cosmic rays like exploding supernovae. On the contrary, positrons and antiprotons are assumed to be secondary cosmic rays, i.e. coming from the interaction of primary cosmic rays with the interstellar media. In addition, electrons and positrons have much smaller mass than protons and antiprotons so they lose much more energy in the galactic magnetic fields. This is illustrated in Fig. 7, where the rigidity dependences of the cosmic ray elementary particles: antiprotons, protons, positrons, and electrons spectrum are compared. As expected, in the rigidity region free of solar modulation effects, above $20 \mathrm{GeV}$, the proton spectrum is much harder that the electron spectrum. However, further studies by AMS bring a lot of surprises. As seen, the behavior of antiprotons (assumed to be secondaries) and protons (assumed to be primaries) at high rigidities are very similar, while the behavior of electrons (i.e. primaries) and positrons (secondaries) are very different. Most surprising is that above $60 \mathrm{GeV}$, positrons, protons and antiprotons display identical an energy dependence whereas electrons exhibit a totally different energy dependence [12] as shown in Fig. 7. The physics explanation for that is yet to be found.

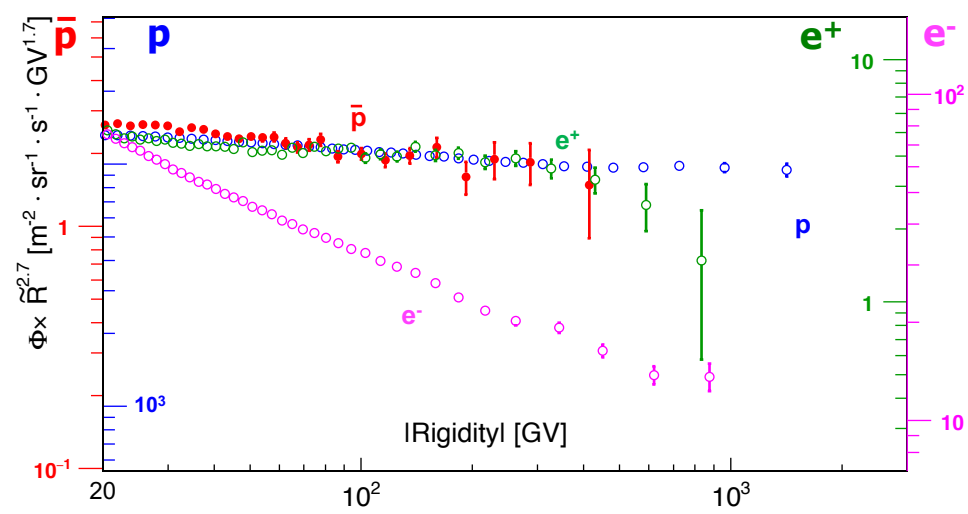

Figure 7: Comparison of the fluxes of elementary particle fluxes (antiprotons, protons, positrons, and electrons) measured by AMS. The positron, antiproton and proton fluxes show the very same functional behavior in the absolute rigidity range $60-500 \mathrm{GV}$, whereas electrons exhibit distinctly different dependence. However, the similarity between positrons and protons/antiprotons deteriorates noticeably at higher energies.

\section{Properties of fluxes of primary and secondary nuclei}

The AMS detector comprises seven instruments, which independently identify different elementary particles as well as nuclei. Protons, helium, lithium, beryllium, boron, carbon, oxygen and heavier nuclei up to iron are intensively studied by AMS (see Fig. 8).

It is assumed that protons, helium, carbon and oxygen are produced directly by primary sources in supernova remnants whereas lithium, beryllium and boron are produced by collisions of primary cosmic rays with the interstellar medium. Primary cosmic rays carry information about their original spectra and propagation, and secondary cosmic rays carry information about the propagation of primary and secondary cosmic rays and the interstellar medium.

High statistics, precision measurements of the helium, carbon, and oxygen fluxes [18] from $2 \mathrm{GV}$ to $3 \mathrm{TV}$, show that these fluxes all deviate from a single power law. Their spectral indices 


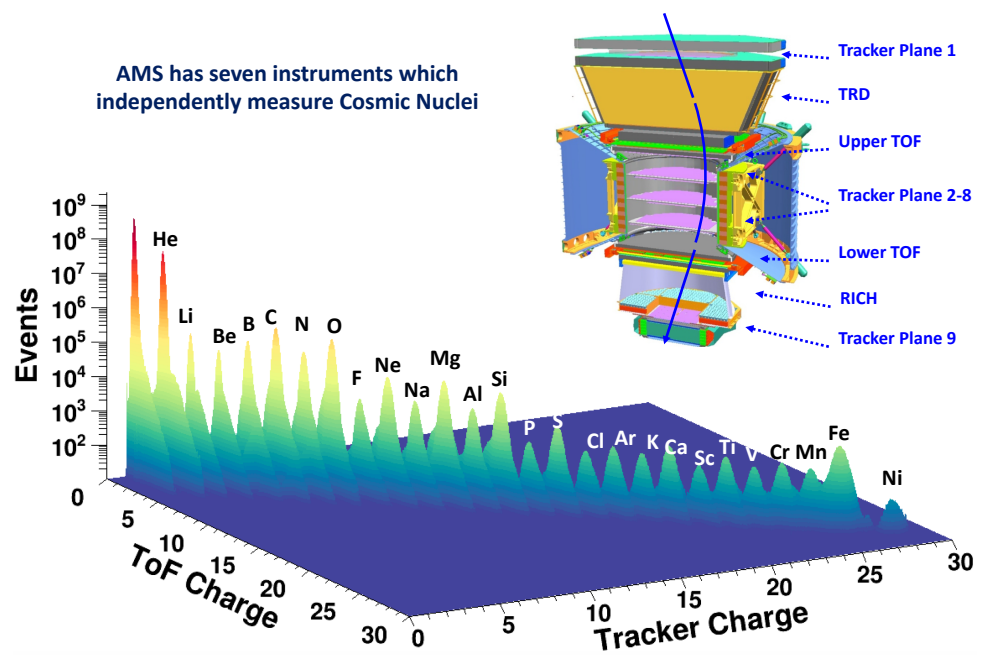

Figure 8: Cosmic ray nuclei measured by AMS. As seen AMS will explore high-Z nuclei beyond Fe.

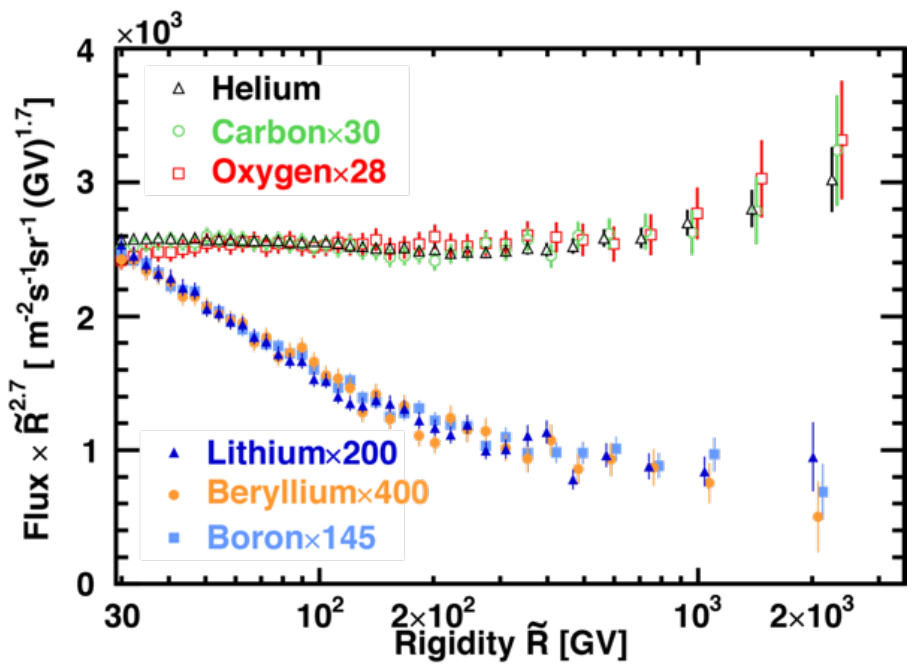

Figure 9: Comparison of the AMS primary [18] and secondary [19] cosmic ray fluxes multiplied by $R^{2.7}$ as a function of rigidity above $30 \mathrm{GV}$. For display purposes, the $\mathrm{C}, \mathrm{O}, \mathrm{Li}, \mathrm{Be}$, and $\mathrm{B}$ fluxes are rescaled as indicated. For clarity, the $\mathrm{He}, \mathrm{O}, \mathrm{Li}$, and $\mathrm{B}$ data points above $400 \mathrm{GV}$ are slightly displaced horizontally. As seen, the three primary fluxes have an identical rigidity dependence above $60 \mathrm{GV}$, as do the three secondary fluxes above $30 \mathrm{GV}$. They all experience hardening above $200 \mathrm{GV}$. However, the rigidity dependences of primary cosmic rays fluxes and of secondary cosmic rays fluxes are distinctly different.

all progressively harden above $200 \mathrm{GV}$. Above $60 \mathrm{GV}$, the three fluxes have identical rigidity dependence, as illustrated in Fig.9. The secondary nuclei fluxes (lithium, beryllium, and boron) [19] also harden above $200 \mathrm{GV}$, but, as seen in Fig.9, their rigidity dependences are distinctly different compared to those of the primary cosmic rays fluxes. AMS performed unique studies in this rigidity range to understand the dynamics of the observed progressive hardening [19]. Figure 10 shows that the ratio of secondary to primary fluxes exhibits a hardening of $0.13 \pm 0.03$.

Precision measurement of the nitrogen flux in cosmic rays [20] from $2.2 \mathrm{GV}$ to $3.3 \mathrm{TV}$ shows 

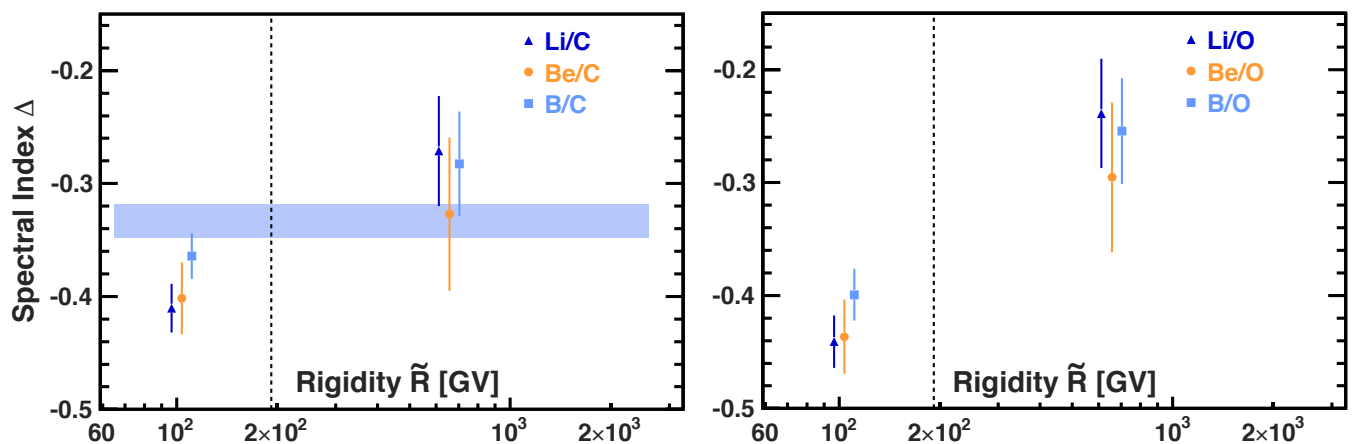

Figure 10: (left plot) The AMS secondary to primary flux ratio spectral indices $\Delta$ as functions of rigidity for $\mathrm{Li} / \mathrm{C}, \mathrm{Be} / \mathrm{C}$, and $\mathrm{B} / \mathrm{C}$. The horizontal band indicates the fit to the $\mathrm{B} / \mathrm{C}$ ratio alone. (right plot) The results for $\mathrm{Li} / \mathrm{O}, \mathrm{Be} / \mathrm{O}$, and $\mathrm{B} / \mathrm{O}$. The vertical dashed line shows the interval boundary. On average, the spectral indices of $\mathrm{Li} / \mathrm{C}, \mathrm{Be} / \mathrm{C}, \mathrm{B} / \mathrm{C}, \mathrm{Li} / \mathrm{O}, \mathrm{Be} / \mathrm{O}$, and $\mathrm{B} / \mathrm{O}$ above $200 \mathrm{GV}$ exhibit a hardening of $0.13 \pm 0.03$ [19] .

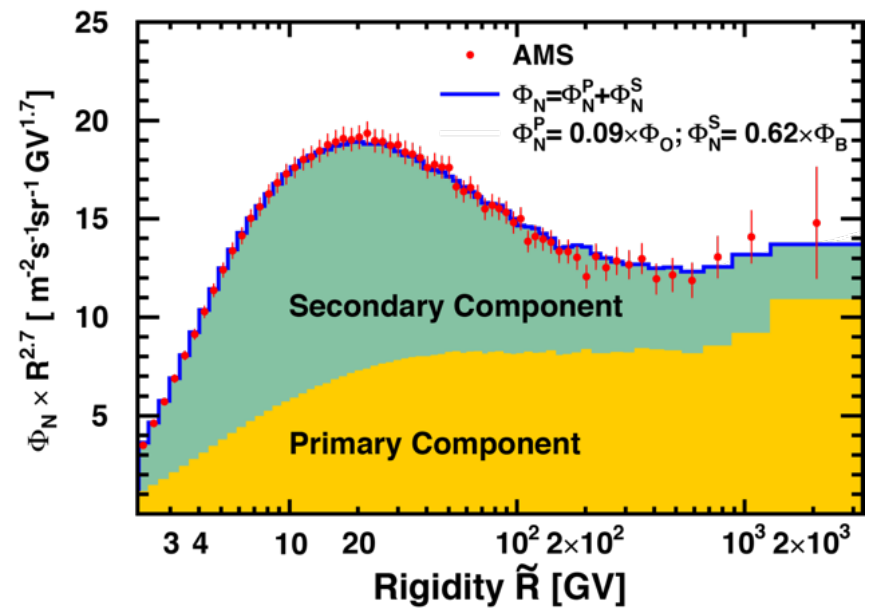

Figure 11: The AMS nitrogen flux $\Phi_{\mathrm{N}}$ fit with the weighted sum of the oxygen flux $\Phi_{\mathrm{O}}$ and the boron flux $\Phi_{\mathrm{B}}$ over the entire rigidity range [20]. The contributions of the primary and secondary components in the nitrogen are indicated by the shading (yellow and green, respectively). The contribution of the secondary component decreases, and the contribution of the primary component increases, with rigidity.

that the nitrogen flux deviates from a single power law. Its spectral index rapidly hardens at high rigidities starting from $\sim 100 \mathrm{GV}$ and becomes identical to the spectral indices of primary cosmic rays $\mathrm{He}, \mathrm{C}$, and $\mathrm{O}$ above $\sim 700 \mathrm{GV}$. Remarkably, the nitrogen flux is well described over the entire rigidity range by the sum of the primary flux $\Phi_{\mathrm{N}}^{\mathrm{p}}$ equal to $9 \%$ of the oxygen flux and the secondary flux $\Phi_{\mathrm{N}}^{\mathrm{s}}$ equal to $62 \%$ of the boron flux as illustrated in Fig. 11. This corresponds to a change of the contribution of the secondary component in the nitrogen flux from $70 \%$ at a few GV to below $30 \%$ above $1 \mathrm{TV}$ [20]. Note that the magnitude of the nitrogen primary component is determined directly at the source, without relying on any propagation models.

\section{Complex Antimatter in Cosmic Rays}

The Big Bang origin of the Universe requires that matter and antimatter be equally abundant at 
the very hot beginning of the universe. The search for the explanation for the absence of antimatter in a complex form is known as Baryogenesis. Baryogenesis requires both a strong symmetry breaking and a finite proton lifetime. Despite the outstanding experimental efforts over the last half a century, no evidence of strong symmetry breaking nor of proton decay have been found. Therefore, the observation of a single anti-helium event in cosmic rays is of great importance.

In seven years of operations, AMS has collected over 120 billion events. From this unparalleled sample of charged cosmic rays 800 million events with charge $|Z|=2$ are selected. All these events have been identified as Helium nuclei, except few that have negative rigidity or $Z=-2$. One of these anti-He candidates is presented in Fig. 12. All of the $\mathrm{Z}=-2$ candidates have one common and peculiar feature - mass around either ${ }^{3} \mathrm{He}$ or ${ }^{4} \mathrm{He}$. At a rate of approximately one antihelium candidate per year and a required signal to background rejection of one in a billion, a detailed understanding of the instrument is required but is exceedingly difficult and time consuming. In the coming years one of our main efforts is to perform stringent detector verification and to collect more data in order to ensure that these $Z=-2$ events are indeed anti-helium.

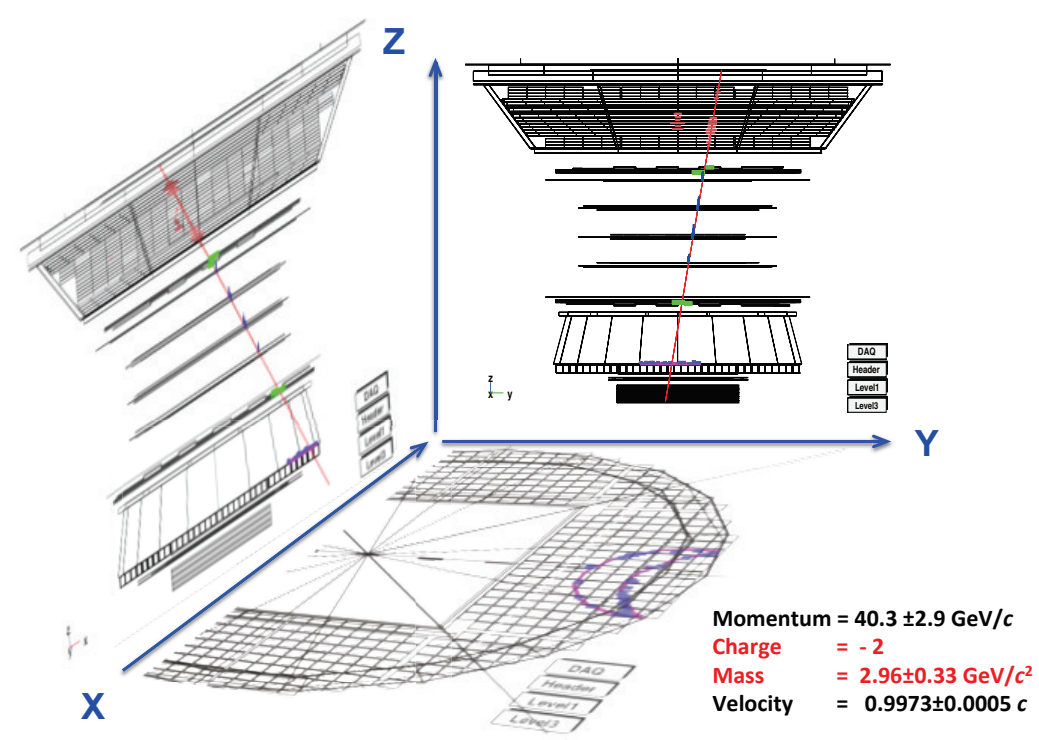

Figure 12: An antihelium candidate with a mass of ${ }^{3} \mathrm{He}$.

\section{Conclusions}

In seven years on the ISS, AMS has recorded more than 120 billion cosmic ray events. The latest AMS measurements of the positron spectrum and positron fraction, the antiproton/proton ratio, the behavior of the fluxes of electrons, positrons, protons, helium and other nuclei provide precise and unexpected information on the production, acceleration and propagation of cosmic rays. The accuracy and characteristics of the data, simultaneously from many different types of cosmic rays require the development of a comprehensive model of cosmic rays.

Of particular significance is our study of complex antimatter in the cosmos. Through stringent detector verification, collecting additional data and anti-deuteron analysis we will ensure that the observed $\mathrm{Z}=-2$ events are indeed anti-helium. 
As a magnetic spectrometer studying cosmic rays, AMS is unique in its precision and energy reach. For the foreseeable future this is the only magnetic spectrometer in space to perform precision measurements and to explore the unknown with high expectations for exciting discoveries.

\section{References}

[1] S.C.C. Ting, Nucl. Phys. B, Proc. Suppl. 243-244 12 (2013); A. Kounine, Int. J. Mod. Phys. E 21123005 (2012).

[2] M. Turner and F. Wilczek, Phys. Rev. D421001 (1990) ; J. Ellis, $26^{\text {th }}$ ICRC Salt Lake City (1999) astro-ph/9911440; H. Cheng, J. Feng and K. Matchev, Phys. Rev. Lett. 89211301 (2002); S. Profumo and P. Ullio, J. Cosmology Astroparticle Phys. JCAP07 006 (2004); D. Hooper and J. Silk, Phys. Rev. D 71083503 (2005); E. Ponton and L. Randall, JHEP 0904080 (2009); D. Hooper, P. Blasi and P. D. Serpico, JCAP 0901025 (2009); Y-Z. Fan et al., Int. J. Mod. Phys. D19 2011 (2010); M. Pato, M. Lattanzi and G. Bertone, JCAP 1012020 (2010).

[3] J. J. Beatty et al., Phys. Rev. Lett. 93241102 (2004); M. Aguilar et al., Phys. Lett. B 646145 (2007); O. Adriani et al., Nature 458607 (2009); O. Adriani et al., Astropart. Phys. 341 (2010); O. Adriani et al., Phys. Rev. Lett. 111081102 (2013); M. Ackermann et al., Phys. Rev. Lett. 108011103 (2012).

[4] Z. Li on behalf of AMS, Presented at this conference, $\mathrm{N}^{\circ} 724$.

[5] M. Aguilar et al., Phys. Rev. Lett. 110, 141102 (2013); L. Accardo et al., Phys. Rev. Lett. 113, 121101 (2014).

[6] Z. Weng on behalf of AMS, Presented at this conference, $N^{\circ} 723$; M. Aguilar et al., Phys. Rev. Lett. 113121102 (2014); M. Aguilar et al., Phys. Rev. Lett. 113221102 (2014).

[7] L. Pacini, on behalf of CALET, Presented at this conference, $\mathrm{N}^{\circ} 463$; A. Tykhonov, on behalf of DAMPE, Presented at this conference, $\mathrm{N}^{\mathrm{o}} 79$.

[8] J. Chang et al., Nature (London) 456, 362 (2008). D. Kerszberg, on behalf of HESS, ICRC-2017, CRI215; M. Ackermann et al., Phys. Rev. D 82, 092004 (2010).

[9] There are many recent excellent theoretical articles on the subject. A few examples are: J. Kopp, Phys. Rev. D 88076013 (2013); Y. Bai et al., Phys. Rev. D 97, 115012 (2018); D. Hooper et al., Phys. Rev. D 96, 103013 (2017); S. Profumo et al., Phys. Rev. D 97, 123008 (2018).

[10] Abeysekara et al., Science 358, 911 (2017).

[11] J. Casaus on behalf of AMS, Presented at this conference, $\mathrm{N}^{\mathrm{o}} 727$.

[12] Z. Tang on behalf of AMS, Presented at this conference, $\mathrm{N}^{\mathrm{o}} 726$;

[13] O. Adriani et al., Phys. Rev. Lett. 102051101 (2009).

[14] K. Abe et al., Phys. Rev. B 670103 (2008).

[15] F. Donato et al., Phys. Rev. Lett. 102071301 (2009).

[16] G.Giesen, et. al., JCAP 09023 (2015); C. Evoli et al., JCAP 12039 (2015); R. Kappl et al., JCAP 10 $03(2015)$;

[17] M.Y. Cui et al., Phys. Rev. Lett. 118191101 (2017); A. Cuoco et al., Phys. Rev. Lett. 118191102 (2017) ; A. Reinert and M.W. Winkler, JCAP 01055 (2018).

[18] Z.Weng on behalf of AMS, Presented at this conference, $\mathrm{N}^{\circ} 720$.

[19] Y. Jia on behalf of AMS, Presented at this conference, $N^{\circ} 721$;

[20] J. Berdugo, on behalf of AMS, Presented at this conference, $\mathrm{N}^{\mathrm{o}} 730$. 